\section{Adaptação dos critérios de avaliação de eventos adversos a medicamentos para uso em um hospital público no Estado do Rio de Janeiro}

\section{Adjustment of evaluation criteria of adverse drug events for use in a public hospital in the State of Rio de Janeiro}

\section{Keroulay Estebanez Roque'}

\section{Enirtes Caetano Prates Melo"}

' Escola de Enfermagem Alfredo Pinto - UNIRIO, RJ e Hospital Universitário Clementino Fraga Filho da Universidade Federal do Rio de Janeiro, RJ.

" Escola Nacional de Saúde Pública - FIOCRUZ; DESP/EEAP da Universidade Federal do Rio de Janeiro, RJ.

\section{Resumo}

Objetivos: Adaptar a metodologia, os critérios e os instrumentos de rastreamento de eventos adversos a medicamentos propostos pelo Institute for Healthcare Improvement e avaliar a ocorrência de eventos adversos a medicamentos em um hospital especializado. Metodologia: $\mathrm{O}$ método selecionado para identificação de eventos adversos a medicamentos foi uma adaptação proposta pelo Institute for Healthcare Improvement, e é baseado na revisão retrospectiva de prontuários orientada por critérios explícitos de rastreamento. O processo de adaptação de critérios de rastreamento de eventos adversos a medicamentos foi realizado por meio de um painel de especialistas com base na análise dos aspectos relacionados à validade, viabilidade e relevância. Essa análise foi realizada em uma amostra de 112 pacientes. Resultados: Foram selecionados 21 critérios de rastreamento para a detecção de eventos adversos a medicamentos. A incidência de eventos adversos a medicamentos no hospital analisado foi de $14,3 \%$. Em $25 \%$ dos casos houve necessidade de intervenção para o suporte de vida. Conclusão: Os critérios de rastreamento de eventos adversos a medicamentos possibilitam um monitoramento dos eventos ao longo do tempo, permitindo avaliar se as estratégias implementadas no sistema de medicação resultaram em melhoria na qualidade da assistência.

Palavras-chave: Eventos adversos. Adaptação de instrumentos. Qualidade da assistência à saúde. Segurança do paciente. 


\section{Abstract}

Objectives: To adapt the methodology, criteria and instruments for tracing adverse drug events proposed by the Institute for Healthcare Improvement and to evaluate the occurrence of adverse drug events in a specialized hospital. Methods: The method selected for the identification of adverse drug events was an adjustment proposed by the Institute for Healthcare Improvement based on the retrospective review of medical charts conducted through explicit tracing criteria. The adjustment process for tracing criteria for adverse drug events was carried out by a panel of specialists based on the analysis of aspects related to validation, viability and relevance. This analysis was carried out from a sample of 112 patients. Results: 21 tracing criteria for the detection of adverse drug events were selected. The incidence of adverse drug events in the analyzed hospital was $14.3 \%$. In $25 \%$ of the cases there was a need for life-support intervention. Conclusion: Tracing criteria for adverse drug events enable monitoring events through time, allowing to evaluate if the strategies implemented in the medication system could show results for a better quality of care.

Keywords: Adverse events. Adjustment of instruments. Quality of health care. Patient safety.

\section{Introdução}

Os eventos adversos relacionados aos processos de cuidar comprometem a segurança do paciente e, muitas vezes, põe sua vida em risco, afetando a qualidade do cuidado. A ocorrência deste tipo de problema reflete o distanciamento entre o cuidado esperado/ideal e o cuidado realizado/ $/$ eal $^{1-2}$.

O cuidado deve ser realizado com qualidade, livre de riscos e falhas, comprometido com a segurança do paciente, promovendo a saúde sem gerar danos ${ }^{3}$. A segurança do paciente tornou-se uma preocupação mundial em função dos efeitos decorrentes de possíveis danos derivados da assistência prestada. Tais conseqüências afetam não somente o paciente, como sua família, os profissionais de saúde envolvidos na assistência, a instituição e a sociedade.

$\mathrm{Na}$ área da saúde, os eventos adversos são comumente associados ao desprestígio profissional, à autopunição e às possíveis sanções. Sendo assim, admitir e relatar o evento não são tarefas triviais ${ }^{3}$. No entanto, a identificação de eventos adversos contribui para o dimensionamento dos problemas ocorridos nos processos de cuidar e é informação valiosa para avaliar a segurança do paciente e a qualidade do cuidado prestado. Para fins deste estudo, evento adverso a medicamento foi considerado como qualquer ocorrência médica desfavorável, que pode acontecer durante o tratamento com um medicamento, mas que não possui, necessariamente, relação causal com esse tratamento ${ }^{4}$.

Não há como negar que o desenvolvimento e a utilização de novas tecnologias diagnósticas e terapêuticas na assistência promovem, a princípio, melhorias na qualidade da assistência e, indiretamente, o aumento da expectativa de vida. No entanto, à medida em que essas inovações tornam-se mais complexas, aumenta a possibilidade de ocorrência desses eventos, o que gera a necessidade de reformulações das mesmas. Estratégias devem ser implementadas para impedir sua ocorrência, baseadas principalmente na educação dos profissionais e 
na mudança cultural da instituição frente ao evento adverso, promovendo assim a qualidade do sistema de saúde e a segurança do paciente ${ }^{2,5,6}$.

Pesquisas sobre o tema segurança do paciente foram realizadas pela Organização Mundial de Saúde (OMS), a Join Commission on Accreditation of Healthcare Organizations (JCAHO), o Institute of Medicine (IOM), o Institute for Healthcare Improvement (IHI), a National Coordinating Council for Medication Error Reporting and Prevention (NCC MERP) e a American Society of Healthy-System Pharmacists (ASHSP) ${ }^{4-12}$. A partir de 1974, estudos realizados na Califórnia, Nova York, Utah, Colorado, Austrália, Nova Zelândia, Inglaterra, Canadá e Estados Unidos da América buscaram identificar a ocorrência dos eventos adversos a medicamentos através da revisão retrospectiva de prontuários em hospitais e identificaram sua alta incidência ${ }^{4-12}$.

Diante da preocupação com a segurança do paciente e a qualidade da assistência prestada, a Agência Nacional de Vigilância Sanitária (ANVISA) implementou no Brasil, em 2001, o projeto Hospitais Sentinelas. Esse projeto favoreceu a integração da área de vigilância em eventos adversos e queixas técnicas da ANVISA com hospitais, hemocentros e serviços de apoio diagnóstico e terapêutico. Seu objetivo foi a construção de uma rede de hospitais em todo país preparada para notificar eventos adversos e queixas técnicas de produtos de saúde, e assim garantir melhores produtos no mercado e mais segurança e qualidade para pacientes e profissionais de saúde ${ }^{13}$. A rede sentinela representa no âmbito da farmacovigilância uma estratégia para integrar a monitoramento de medicamentos à prática clínica, realizando atividades relativas à identificação, avaliação, compreensão e prevenção de efeitos adversos ou qualquer problema possível relacionado com medicamentos ${ }^{13}$.

Considerando que a terapia medicamentosa é amplamente utilizada como modalidade de tratamento de pacientes com diversas alterações de saúde, a identificação de eventos adversos a medicamentos ganha destaque, tendo como meta a segurança do paciente e a qualidade do cuidado em saúde. Para tanto é necessária a consolidação de sistemas de vigilância capazes de rastrear situações de risco e possíveis eventos adversos a medicamentos, instituindo entre os profissionais, usuários e gestores de saúde uma atitude de constante vigilância.

Este estudo teve como objetivo adaptar a metodologia, os critérios e os instrumentos de rastreamento de eventos adversos a medicamentos proposto pelo Institute for Healthcare Improvement e avaliar a ocorrência de eventos adversos a medicamentos em um hospital especializado.

\section{Metodologia}

A metodologia selecionada para identificação de eventos adversos a medicamentos foi uma adaptação dos critérios e instrumentos de rastreamento daqueles propostos pelo $\mathrm{IHI}^{11}$. Trata-se de um estudo que vem incorporar avanços sugeridos em pesquisas antecedentes e que fornece um método cuja aplicação apresenta um grau de dificuldade relativamente baixo. Esta metodologia, baseada em uma revisão retrospectiva de prontuários orientada por critérios explícitos de rastreamento, fornece boas indicações da natureza e da incidência dos eventos adversos. No entanto, a disponibilidade de dados confiáveis e a qualidade das informações registradas nos prontuários podem comprometer a detecção do evento adverso.

A pesquisa foi desenvolvida em duas etapas, que envolveram a adaptação dos critérios de rastreamento de eventos adversos a medicamentos e a avaliação da ocorrência de eventos em um hospital público, localizado no Município do Rio de Janeiro.

\section{Primeira etapa: adaptação dos critérios de rastreamento de eventos adversos a medicamentos}

Para adaptar os critérios de rastreamento de eventos adversos a medicamentos estabelecidos a partir da metodologia propos- 
ta pelo IHI, utilizou-se o método de Delphi modificado ${ }^{14}$ para obter consenso no painel de especialistas. Trata-se de um método de estruturação sistemática de processos de julgamentos e confronto sobre um tópico particular em um grupo de indivíduos.

O painel de especialistas foi composto por 10 profissionais da área da saúde (4 enfermeiros, 2 farmacologistas, 1 farmacêutico e 3 médicos) selecionados a partir de vasta experiência nas respectivas áreas de atuação. Esses profissionais possuem mais de 10 anos de experiência em suas especialidades, sendo 4 doutores, 3 mestres e 3 especialistas. Os especialistas, após confirmarem sua participação, receberam as informações por escrito referentes à pesquisa e à metodologia do estudo e uma ficha de avaliação dos critérios propostos. Os painelistas não foram remunerados.

O processo de avaliação dos critérios de rastreamento de eventos adversos a medicamentos foi realizado em 2 fases. Na primeira foram submetidos 25 critérios (Quadro 1). Os painelistas opinaram por escrito a respeito dos critérios de rastreamento, através de um roteiro, de forma que nenhum dos outros participantes conhecesse suas opiniões.

Três aspectos foram avaliados pelos especialistas: viabilidade, validade e relevância dos rastreadores. A viabilidade referiase à facilidade operacional, aos custos, à disponibilidade de dados necessários e a outras barreiras potencialmente associadas à aplicação do rastreador. A validade diz respeito ao grau com que o rastreador alcança seus objetivos, ou seja, se reflete o evento ou o aspecto que se propõe a medir. A relevância esteve associada à aplicabilidade e à pertinência do rastreador.

Durante o processo de avaliação, cada especialista julgou a pertinência dos critérios de rastreamento adaptados e atribuiu pontos utilizando uma escala de valor numérico que variava de 1 a 9. Um ponto corresponderia a uma avaliação extremamente negativa, e nove a uma avaliação extremamente positiva. Aos escores situados entre 1 e 6 solicitou-se justificativa.
A seguir procedeu-se à decisão final dos critérios a serem aplicados no estudo e a síntese do processo foi encaminhada aos especialistas.

\section{Segunda etapa: avaliação da ocorrência de eventos adversos a medicamentos}

Na segunda etapa do estudo procedeuse à análise retrospectiva de prontuários para identificação de eventos adversos a medicamentos, utilizando-se adaptação da metodologia proposta pelo $\mathrm{IHI}^{11}$. O universo do estudo foi composto por pacientes internados no período de dezembro de 2007 a fevereiro de $2008 \mathrm{em}$ um hospital de grande porte - Hospital Y, especializado na área de cardiologia. Foram considerados elegíveis pacientes maiores de 18 anos que permaneceram internados por pelo menos 48 horas. Foram excluídos pacientes provenientes da obstetrícia e aqueles que estavam em tratamento oncológico.

Foram elegíveis para o estudo 551 prontuários, sendo extraída uma amostra aleatória simples de 112 prontuários, estimada a partir dos seguintes critérios: erro de $10 \%$, significância estatística de 95\%, prevalência do evento estudado de $10 \%$ e perdas de $10 \%$. A seleção aleatória dos prontuários foi realizada a partir da listagem mensal de alta, obtida junto ao Serviço de Admissão e Alta do hospital.

Readmissões hospitalares ocorridas nos primeiros 30 dias, por razões relacionadas ao diagnóstico que motivou a internação, foram consideradas reinternações. Esses casos foram considerados como uma única internação.

Para esse estudo foi desenvolvido um programa computacional de eventos adversos a medicamento, destinado ao armazenamento de dados relativos ao rastreamento e à avaliação de eventos. Os critérios rastreadores utilizados (Quadro 1), adaptados da lista original, foram investigados no sumário de alta, nos exames laboratoriais, nas prescrições de medicamentos e nas evoluções da equipe de profissionais.

$\mathrm{Na}$ etapa inicial de coleta dos dados 
Quadro 1 - Adaptação dos critérios de rastreamento de eventos adversos a medicamentos propostos pelo Institute for Healthcare Improvement $(\mathrm{IHI})$.

Chart 1 - Adjustment of criteria for tracing adverse drug events proposed by the Institute for Healthcare Improvement (IHI).

\begin{tabular}{|c|c|c|}
\hline $\begin{array}{l}\text { Critério de rastreamento de eventos } \\
\text { adversos a medicamentos propostos } \\
\text { pelo } \mathrm{IHI}\end{array}$ & $\begin{array}{l}\text { Critérios de rastreamento de eventos } \\
\text { adversos a medicamentos submetidos } \\
\text { ao consenso de especialistas }\end{array}$ & $\begin{array}{l}\text { Decisão adotada após adaptação por } \\
\text { painel de especialistas }\end{array}$ \\
\hline 1. difenidramina & 1. difenidramina & 1. alterado - anti-histamínicos \\
\hline 2. vitamina $\mathrm{K}$ & 2. vitamina $\mathrm{K}$ & 2. mantido critério do IHI \\
\hline 3. flumazenil & 3. flumazenil & 3. mantido critério do IHI \\
\hline 4. droperidol & 4. droperidol & 4. alterado-antieméticos \\
\hline 5. naloxona & 5. naloxona & 5. mantido critério do $\mathrm{IHI}$ \\
\hline 6. antidiarréicos & 6. antidiarréicos & 6. mantido critério do IHI \\
\hline 7. poliestireno de sódio & sódio & 7. excluído \\
\hline $\begin{array}{l}\text { 8. tempo de protrombina }(\mathrm{PTT})>100 \\
\text { segundos }\end{array}$ & $\begin{array}{l}\text { 8. tempo de protrombina }(\mathrm{PTT})>100 \\
\text { segundos }\end{array}$ & 8. mantido critério do $\mathrm{IHI}$ \\
\hline 9. INR > 6 & 9. INR $>6$ & 9. mantido critério do IHI \\
\hline 10. neutrófilos $<3000 \times 10^{6} / \mu \mathrm{L}$ & 10. neutrófilos $<3000 \times 10^{6} / \mu \mathrm{L}$ & 10. mantido critério do IHI \\
\hline 11. glicose sérica $<50 \mathrm{mg} / \mathrm{dL}$ & 11. glicose sérica $<50 \mathrm{mg} / \mathrm{dL}$ & $\begin{array}{l}\text { 11. alterado- glicose sérica ou capilar } \\
<50 \mathrm{mg} / \mathrm{dL}\end{array}$ \\
\hline 12. aumento sérico de creatinina & 12. aumento sérico de creatinina & $\begin{array}{l}\text { 12. alterado - creatinina sérica > 1,5 } \\
\mathrm{mg} / \mathrm{dL}\end{array}$ \\
\hline 13. Clostridium difficile positivo & 13. Clostridium difficile positivo & 13. excluído \\
\hline 14. nível de digoxina $>2 \mathrm{ng} / \mathrm{mL}$ & 14. nível de digoxina $>2 \mathrm{ng} / \mathrm{mL}$ & $\begin{array}{l}\text { 14. alterado - nível de digoxina }>2 \\
\mathrm{mcg} / \mathrm{dl} \text { e uso de digoxina }\end{array}$ \\
\hline 15. nível de lidocaína $>5$ । & 15. nível de lidocaína $>5 \mathrm{ng} / \mathrm{mL}$ & 15. excluído \\
\hline $\begin{array}{l}\text { 16. nível de gentamicina ou } \\
\text { tobramicina }>10 \mu \mathrm{g} / \mathrm{ml} \mathrm{a} 2 \mu \mathrm{g} / \mathrm{mL}\end{array}$ & $\begin{array}{l}\text { 16. nível de gentamicina ou } \\
\text { tobramicina }>2 \mu \mathrm{g} / \mathrm{ml} \mathrm{a} 10 \mu \mathrm{g} / \mathrm{mL}\end{array}$ & $\begin{array}{l}\text { 16. alterado - uso de gentamicina ou } \\
\text { tobramicina }\end{array}$ \\
\hline $\begin{array}{l}\text { 17. nível de amicacina }>30 \mu \mathrm{g} / \mathrm{mL} \text { a } \\
>10 \mu \mathrm{g} / \mathrm{mL}\end{array}$ & $\begin{array}{l}\text { 17. nível de amicacina }>10 \mu \mathrm{g} / \mathrm{mL} \text { a } \\
>30 \mu \mathrm{g} / \mathrm{mL}\end{array}$ & 17. alterado - uso de amicacina \\
\hline 18. nível de vancomicina $>26 \mu \mathrm{g} / \mathrm{mL}$ & 18. nível de vancomicina $>26 \mu \mathrm{g} / \mathrm{mL}$ & 18. alterado - uso de vancomicina \\
\hline 19. nível de teofilina $>20 \mu \mathrm{g} / \mathrm{mL}$ & 19. nível de teofilina $>20 \mu \mathrm{g} / \mathrm{mL}$ & 19. excluído \\
\hline 20. sedação, letargia e queda & 20. sedação, letargia e queda & 20. mantido critério do IHI \\
\hline 21. rash & 21. rash & 21. mantido critério do IHI \\
\hline 22. retirada abrupta do medicamento & 22. retirada abrupta do medicamento & 22. mantido critério do $\mathrm{IHI}$ \\
\hline $\begin{array}{l}\text { 23. transferência para Unidade de } \\
\text { Terapia Intensiva ou Semi-Intensiva }\end{array}$ & $\begin{array}{l}\text { 23. transferência para Unidade de } \\
\text { Terapia Intensiva ou Semi-Intensiva }\end{array}$ & 23. mantido critério do IHI \\
\hline $\begin{array}{l}\text { 24. rastreador específico para a } \\
\text { instituição }\end{array}$ & 24. sulfato de protamina & 24. mantido critério após consenso \\
\hline 25. ----- & 25. glicose hipertônica a $25 \%$ ou $50 \%$ & 25. mantido critério após consenso \\
\hline
\end{tabular}

adotou-se procedimento de dupla revisão de prontuários, baseada em critérios explícitos, por duas enfermeiras revisoras independentes. A presença de pelo menos um critério garantia a seleção do prontuário para a segunda etapa do processo de avaliação. Na etapa seguinte, um médico, um farmacêutico e uma enfermeira avaliaram a ocorrência dos eventos adversos a medicamentos detectados na primeira etapa, através de uma avaliação implícita. A confirmação do evento adverso ocorreu através da estratégia de consenso destes especialistas. Uma vez identificado um possível evento adverso a medicamentos, sinalizado por um critério de rastreamento, foi realizada uma nova avaliação deste prontuário com a finalidade de confirmar a ocorrência do evento através de evidências clínicas e da cadeia de causalidade do evento, determinando assim se o evento ocorreu antes, durante ou após administração do medicamento. As divergências foram resolvidas por consenso a partir do julgamento clínico. 
O projeto de pesquisa foi submetido e aprovado pelo Comitê de Ética e Pesquisa do hospital estudado, com o parecer $\mathrm{n}^{\circ}$ 0192/22.02.2008. Procedimentos para impossibilitar a identificação do indivíduo e para a manutenção da confidencialidade dos dados individuais dos pacientes foram adotados. Não há conflito de interesses no estudo.

\section{Resultados}

Dos 25 critérios de rastreamento de eventos adversos a medicamentos submetidos ao painel de especialistas, 4 critérios foram excluídos e 8 alterados, totalizando 21 critérios selecionados para a detecção de eventos adversos (Quadro 1). Destes critérios, 12 estão relacionados ao uso de medicamentos, 5 às alterações nos exames laboratoriais que possam ser associados ao uso de medicamentos e 4 aos possíveis sinais e sintomas apresentados pelos pacientes após o uso de determinado medicamento registrados pelos profissionais de saúde (Quadro 1).

Os critérios relacionados ao uso de "poliestireno de sódio" (média do escore 21,1; desvio-padrão \pm 8 ), à presença de "Clostridium difficile positivo" em cultura (média do escore 12,8; desvio-padrão $\pm 10,4$ ) e à avaliação do "nível de lidocaína > 5ng/ml" (média do escore 17,4; desvio-padrão $\pm 7,3$ ) e "nível de teofilina $>20 \mu \mathrm{g} / \mathrm{ml}$ " (média do escore 20,1; desvio-padrão $\pm 7,7$ ) obtiveram na avaliação dos especialistas os mais baixos escores em relação às dimensões estudadas (validade, viabilidade e relevância) e foram excluídos. O valor máximo obtido em cada escore foi 27 e o mínimo 3. No hospital estudado não são realizados os exames para a detecção de Clostridium difficile e a mensuração do nível de lidocaína e teofilina sérica (Quadro 1).

Segundo o IHI, o método de rastreamento de eventos adversos a medicamentos possui 24 critérios, sendo que o último critério é descrito como um "rastreador específico para a instituição" e, portanto, de inclusão opcional. Para fins desse estudo, o critério "sulfato de protamina" foi considerado aquele específico da instituição e obteve escore elevado pela avaliação dos especialistas (média 26,2, desvio-padrão \pm $3,5)$. O uso do critério "sulfato de protamina" justifica-se pela possibilidade de detecção de eventos relacionados à alteração da coagulação sanguínea, geralmente sangramentos, decorrentes do uso de heparina ${ }^{15}$, anticoagulante injetável usado em larga escala no hospital estudado. O critério "glicose hipertônica $25 \%$ ou $50 \%$ " foi incluído pelos pesquisadores nesse estudo e, submetido à avaliação pelo consenso de especialistas, obteve escore elevado (média 25,5 , desvio-padrão $\pm 3,8$ ). O critério "glicose hipertônica $25 \%$ ou $50 \%$ " favorece a identificação de situações em que o paciente apresenta sinais e sintomas de hipoglicemia sem apresentar alteração brusca da glicose sérica (glicose $<50 \mathrm{mg} / \mathrm{dl}$ ), exigindo assim a sua administração ${ }^{16}$.

No estudo foram realizadas alterações nos seguintes critérios propostos pelo $\mathrm{IHI}^{11}$ : "difenidramina", "droperidol”, avaliação de "glicose sérica $<50 \mathrm{mg} / \mathrm{dl}$, "aumento de creatinina sérica", "nível de digoxina $>2 \mathrm{ng} / \mathrm{ml}$ ", "nível de gentamicina ou tobramicina entre $>2 \mu \mathrm{g} / \mathrm{ml}$ a $>10 \mu \mathrm{g} / \mathrm{ml}$ ", "nível de amicacina entre $>10 \mu \mathrm{g} / \mathrm{ml}$ a > $30 \mu \mathrm{g} / \mathrm{ml}$ " e "nível de vancomicina $>26 \mu \mathrm{g} / \mathrm{ml}$ ". As adaptações sugeridas pelo painel de especialistas visaram tornar os critérios mais precisos e adequados ao perfil do hospital estudado (Quadro 1). Adicionalmente, considerando a realidade brasileira, o critério de rastreamento foi modificado para "glicose sérica ou capilar < $50 \mathrm{mg} / \mathrm{dl}$ ” e o processo de identificação foi alterado para hipoglicemia relacionada ao uso de insulina ou hipoglicemiantes orais (Quadro 1).

O painel de especialistas julgou pertinente a alteração do critério uso de "difenidramina" e de "droperidol" por uso de "anti-histamínicos" e "antieméticos", respectivamente, uma vez que, no Brasil, existem outros antihistamínicos e antieméticos prescritos nas instituições de saúde (Quadro 1).

Os especialistas propuseram alteração do parâmetro "aumento de creatinina séri- 
ca" para "creatinina sérica $>1,5 \mathrm{mg} / \mathrm{dl}$ " a fim de tornar o critério mais preciso e específico. A elevação do nível sérico de creatinina pode estar relacionada à insuficiência renal ocasionada por administração determinado medicamento (Quadro 1).

Eventos de intoxicação digitálica foram identificados a partir do "nível de digoxina sérica" e através de sinais e sintomas de intoxicação digitálica (Quadro 1). O nível de digoxina considerado tóxico nesse estudo foi $>2 \mathrm{mcg} / \mathrm{dl}$. Tendo em conta que o laboratório da instituição estudada utiliza a medida de mcg/dl no teste de diagnóstico de intoxicação por digitálicos foi necessário o ajuste da unidade de medida.

Apesar da alta relevância dada à avaliação dos níveis séricos de gentamicina, tobramicina, amicacina e vancomicina, relacionado ao risco de ototoxicidade e nefrotoxicidade desses antibióticos, o painel de especialistas sinalizou sua baixa viabilidade, uma vez que esta avaliação não constitui uma rotina na instituição hospitalar estudada. Os critérios relacionados com os níveis séricos desses antibióticos foram modificados para "uso de gentamicina ou tobramicina", "uso de amicacina" e "uso de vancomicina" (Quadro 1).

Os escores mais elevados na avaliação dos especialistas foram dados ao uso de “vitamina K” (média 24,6; desvio-padrão $\pm 3,5$ ), uso de "flumazenil" (média 25,7; desvio-padrão $\pm 2,1$ ), uso de "naloxona" (média 25,7; desvio-padrão $\pm 2,2$ ), uso de "glicose hipertônica $25 \%$ ou 50\%" (média 25,5; desvio-padrão $\pm 3,8$ ) e a avaliação da coagulação sanguínea através do "tempo de tromboplastina parcial” (PTT) (média 26,1; desvio-padrão $\pm 2,0$ ) e da "norma reguladora internacional” (INR) (média 26,7; desviopadrão $\pm 0,95$ ).

Foram avaliados 112 prontuários elegíveis dentre aqueles pacientes internados no período estudado. Nesses prontuários foram rastreados 238 critérios relacionados à possibilidade de ocorrência de eventos adversos. Entre os casos, verificou-se uma pequena predominância do sexo feminino (57,9\%) e uma média de idade de 59 anos (desvio-padrão \pm 15,82). Após avaliação, confirmaram-se 16 eventos adversos a medicamento, correspondendo a uma incidência de 14,3\% (Tabela 1).

Tabela 1 - Casos de eventos adversos a medicamentos, Hospital Y, Rio de Janeiro, dezembro de 2007 a fevereiro de 2008. Table 1 - Cases of adverse drug events, Hospital Y, Rio de Janeiro, from December 2007 to February 2008.

\begin{tabular}{|c|c|c|c|c|}
\hline $\begin{array}{l}\text { Características do } \\
\text { paciente } \\
\text { (idade, sexo e } \\
\text { diagnóstico) }\end{array}$ & $\begin{array}{l}\text { No de eventos } \\
\text { adversos a } \\
\text { medicamentos }\end{array}$ & $\begin{array}{c}\text { Descrição de } \\
\text { eventos adversos a } \\
\text { medicamentos }\end{array}$ & $\begin{array}{l}\text { Medicamentos } \\
\text { Suspeitos }\end{array}$ & $\begin{array}{l}\text { Critérios de rastreamento } \\
\text { relacionado com evento } \\
\text { adverso a medicamentos }\end{array}$ \\
\hline $\begin{array}{l}39 \text { anos, feminino, } \\
\text { Estenose de Mitral }\end{array}$ & 1 & $\begin{array}{l}\text { Prurido em membros } \\
\text { superiores e dorso }\end{array}$ & contraste radiológico & anti-histamínico \\
\hline $\begin{array}{l}76 \text { anos, masculino, } \\
\text { Bloqueio Átrio } \\
\text { Ventricular Total }\end{array}$ & 1 & Hematúria & $\begin{array}{l}\text { enoxaparina e ácido } \\
\text { acetilsalicílico }\end{array}$ & $\begin{array}{l}\text { retirada abrupta do } \\
\text { medicamento }\end{array}$ \\
\hline $\begin{array}{l}65 \text { anos, masculino, } \\
\text { Infarto Agudo do } \\
\text { Miocárdio }\end{array}$ & 1 & Hematúria & $\begin{array}{c}\text { enoxaparina, } \\
\text { clopidrogel e ácido } \\
\text { acetilsalicílico }\end{array}$ & $\begin{array}{l}\text { retirada abrupta do } \\
\text { medicamento }\end{array}$ \\
\hline $\begin{array}{l}58 \text { anos, feminino, } \\
\text { Estenose Aórtica } \\
\text { Reumática }\end{array}$ & 1 & $\begin{array}{l}\text { Hematoma } \\
\text { generalizado }\end{array}$ & varfarina & $\begin{array}{l}\text { vitamina K } \\
\text { INR } \\
\text { retirada abrupta de } \\
\text { medicamento }\end{array}$ \\
\hline $\begin{array}{l}34 \text { anos, masculino, } \\
\text { Infarto Agudo do } \\
\text { Miocárdio }\end{array}$ & 1 & $\begin{array}{l}\text { Hematoma em região } \\
\text { coxo femural direita }\end{array}$ & $\begin{array}{c}\text { tirofiban, clopidrogel e } \\
\text { ácido acetilsalicílico }\end{array}$ & $\begin{array}{l}\text { PTT }>100 \text { segundos } \\
\text { retirada abrupta de } \\
\text { medicamento }\end{array}$ \\
\hline
\end{tabular}


Tabela 1 - Casos de eventos adversos a medicamentos, Hospital Y, Rio de Janeiro, dezembro de 2007 a fevereiro de 2008. (continuação)

Table 1 - Cases of adverse drug events, Hospital Y, Rio de Janeiro, from December 2007 to February 2008. (continuation)

\begin{tabular}{|c|c|c|c|c|}
\hline $\begin{array}{l}\text { Características do } \\
\text { paciente } \\
\text { (idade, sexo e } \\
\text { diagnóstico) }\end{array}$ & $\begin{array}{l}N^{\circ} \text { de eventos } \\
\text { adversos a } \\
\text { medicamentos }\end{array}$ & $\begin{array}{l}\text { Descrição de } \\
\text { eventos adversos a } \\
\text { medicamentos }\end{array}$ & $\begin{array}{l}\text { Medicamentos } \\
\text { Suspeitos }\end{array}$ & $\begin{array}{l}\text { Critérios de rastreamento } \\
\text { relacionado com evento } \\
\text { adverso a medicamentos }\end{array}$ \\
\hline $\begin{array}{l}24 \text { anos, masculino, } \\
\text { Aneurisma de Aorta } \\
\text { Ascendente }\end{array}$ & 1 & $\begin{array}{l}\text { Hemorragia em } \\
\text { pericárdio }\end{array}$ & $\begin{array}{c}\text { enoxaparina e } \\
\text { varfarina }\end{array}$ & $\begin{array}{l}\text { INR } \\
\text { retirada abrupta de } \\
\text { medicamento } \\
\text { tranferência para UTI ou } \\
\text { Semi-Intensiva }\end{array}$ \\
\hline $\begin{array}{l}57 \text { anos, feminino, } \\
\text { Estenose Mitral }\end{array}$ & 1 & $\begin{array}{c}\text { Hemorragia no pós- } \\
\text { operatório de troca de } \\
\text { valva mitral }\end{array}$ & heparina & sulfato de protamina \\
\hline $\begin{array}{l}52 \text { anos, masculino, } \\
\text { Fluter Atrial }\end{array}$ & 1 & $\begin{array}{l}\text { Hemorragia após } \\
\text { a circulação extra- } \\
\text { corpórea }\end{array}$ & heparina & sulfato de protamina \\
\hline $\begin{array}{l}72 \text { anos, masculino, } \\
\text { Estenose de Aorta }\end{array}$ & 2 & $\begin{array}{l}\text { Hipoglicemia e } \\
\text { Lesão renal }\end{array}$ & $\begin{array}{l}\text { glibenclamida, } \\
\text { metformina e } \\
\text { espirolactona }\end{array}$ & $\begin{array}{l}\text { glicose hipertônica } 25 \% \\
\text { ou } 50 \% \\
\text { glicemia sérica ou capilar } \\
<50 \mathrm{mg} / \mathrm{dl} \\
\text { creatinina }>1,5 \mathrm{mg} / \mathrm{dl} \\
\text { retirada abrupta de } \\
\text { medicamento }\end{array}$ \\
\hline $\begin{array}{l}59 \text { anos, masculino, } \\
\text { Angina Instável }\end{array}$ & 1 & Hipoglicemia & glibenclamida & $\begin{array}{l}\text { glicemia sérica ou capilar } \\
<50 \mathrm{mg} / \mathrm{dl}\end{array}$ \\
\hline $\begin{array}{l}70 \text { anos, feminino, } \\
\text { Infarto Agudo do } \\
\text { Miocárdio }\end{array}$ & 1 & Hipoglicemia & Insulina & $\begin{array}{l}\text { glicose hipertônica } 25 \% \\
\text { ou } 50 \% \\
\text { glicemia sérica ou capilar } \\
<50 \mathrm{mg} / \mathrm{dl}\end{array}$ \\
\hline $\begin{array}{l}60 \text { anos, masculino, } \\
\text { Angina Estável }\end{array}$ & 1 & Hipoglicemia & Insulina & $\begin{array}{l}\text { glicose hipertônica } 25 \% \\
\text { ou } 50 \% \\
\text { glicemia sérica ou capilar } \\
<50 \text { mg/dl }\end{array}$ \\
\hline $\begin{array}{l}79 \text { anos, masculino, } \\
\text { Infarto Agudo do } \\
\text { Miocárdio }\end{array}$ & 1 & Hipoglicemia & Insulina & $\begin{array}{l}\text { glicose hipertônica } 25 \% \\
\text { ou } 50 \% \\
\text { glicemia sérica ou capilar } \\
<50 \mathrm{mg} / \mathrm{dl}\end{array}$ \\
\hline $\begin{array}{l}84 \text { anos, masculino, } \\
\text { Insuficiência Cardíaca }\end{array}$ & 1 & Bradicardia & digoxina & $\begin{array}{l}\text { nível de digoxina }>2 \mathrm{mcg} / \mathrm{dl} \\
\text { e uso de digoxia } \\
\text { Retirada abrupta de } \\
\text { medicamento }\end{array}$ \\
\hline $\begin{array}{l}73 \text { anos, feminino, } \\
\text { Insuficiência Cardíaca }\end{array}$ & 1 & Fibrilação Atrial & digoxina & $\begin{array}{l}\text { nível de digoxina }>2 \mathrm{mcg} / \mathrm{dl} \\
\text { e uso de digoxia } \\
\text { retirada abrupta de } \\
\text { medicamento }\end{array}$ \\
\hline
\end{tabular}


Nos casos estudados foram identificados 4 eventos adversos a medicamentos entre o segundo e o quarto dia de internação e 5 eventos adversos em pacientes com mais de 26 dias de internação hospitalar.

As unidades de cuidados intensivos concentram a maior frequência de eventos adversos a medicamentos (43,7\%), seguidas de enfermaria (25\%) e centro cirúrgico (25\%).

Considerando a contribuição dos critérios de rastreamento na identificação de eventos adversos a medicamentos, a "retirada abrupta do medicamento" ( $\mathrm{n}=50)$, a "transferência do paciente para Unidade de Terapia Intensiva ou Semi-Intensiva" ( $\mathrm{n}=$ 23), a prescrição de "antieméticos" $(n=33)$, de "nível de digoxina > $2 \mathrm{mcg} / \mathrm{dl}$ ou uso de di- goxina" ( $\mathrm{n}=30$ ) e de "sulfato de protamina" $(\mathrm{n}=23)$ foram os critérios identificados com mais frequência. O critério "retirada abrupta do medicamento" detectou sete eventos adversos. A utilização do rastreador "nível de digoxina $>2 \mathrm{mcg} / \mathrm{dl}$ ou uso de digoxina" possibilitou a detecção de dois eventos relacionados à intoxicação por digitálicos. Através do critério "sulfato de protamina" foram identificados dois eventos relacionados à alteração na coagulação sanguínea. A "transferência do paciente para Unidade Terapia Intensiva ou Semi-Intensiva" permitiu a identificação de um evento adverso (Tabela 2). O uso de "antieméticos" não esteve atrelado à ocorrência de eventos adversos identificáveis.

Tabela 2 - Identificação de eventos adversos a medicamentos, Hospital Y, Rio de Janeiro, dezembro de 2007 a fevereiro de 2008.

Table 2 - Identification of adverse drug events, Hospital Y, Rio de Janeiro, from December 2007 to February 2008.

\begin{tabular}{|c|c|c|}
\hline Rastreadores de eventos adversos a medicamentos & $\begin{array}{c}\text { Frequência } \\
\text { n (\%) }\end{array}$ & $\begin{array}{c}\text { Proporção de eventos adversos a } \\
\text { medicamentos detectados segundo } \\
\text { rastreador específico* }\end{array}$ \\
\hline anti-histamínicos & $6(2,5)$ & $16,7(1 / 6)$ \\
\hline vitamina $\mathrm{K}$ & $13(5,5)$ & $7,7(1 / 13)$ \\
\hline sulfato de protamina & $23(9,7)$ & $8,7(2 / 23)$ \\
\hline flumazenil & - & - \\
\hline naloxona & - & - \\
\hline antiemético & $33(13,9)$ & - \\
\hline antidiarréico & $1(0,4)$ & - \\
\hline glicose hipertônica a $25 \%$ ou $50 \%$ & $10(4,2)$ & $40,0(4 / 10)$ \\
\hline nível de digoxina $>2 \mathrm{mcg} / \mathrm{dl}$ e uso de digoxia & $30(12,6)$ & $6,7(2 / 30)$ \\
\hline tempo de protrombina $>100$ segundos & $1(0,4)$ & - \\
\hline INR $>6$ & $7(2,9)$ & $28,6(2 / 7)$ \\
\hline neutrófilos $<3000 \times 10^{6} / \mu \mathrm{l}$ & $1(0,4)$ & - \\
\hline glicose sérica ou capilar < 50mg/dl & $9(3,8)$ & $55,6(5 / 9)$ \\
\hline creatinina $>1,5 \mathrm{mg} / \mathrm{dl}$ & $22(9,2)$ & $4,5(1 / 22)$ \\
\hline nível de tobramicina ou gentamicina & $1(0,4)$ & - \\
\hline nível de amicacina & $1(0,4)$ & - \\
\hline nível de vancomicina & $4(1,7)$ & - \\
\hline sedação, letargia e queda & $3(1,3)$ & - \\
\hline rash & - & - \\
\hline retirada abrupta do medicamento & $50(21,0)$ & $14,0(7 / 50)$ \\
\hline transferência para UTI ou semi-intensiva & $23(9,7)$ & $4,3(1 / 23)$ \\
\hline Total & $238(100,0)$ & - \\
\hline
\end{tabular}

* Número de eventos adversos a medicamentos em relação ao número de vezes que o rastreador específico foi detectado

* [Number of adverse drug events in relation to number of times the specific tracer was detected] 


\section{Discussão}

O sucesso de um estudo de incidência de eventos adversos depende de um método que produza julgamentos válidos $\mathrm{e}$ seguros $^{12}$. De um modo geral, os critérios de rastreamento de eventos adversos possibilitam um monitoramento dos eventos ao longo do tempo, permitindo avaliar se as estratégias implementadas no sistema de medicação resultaram em melhoria na qualidade da assistência, traduzida em segurança para os pacientes e redução de erros de medicação ${ }^{11}$.

A capacidade do instrumento adaptado em discriminar, em uma população sob estudo, a evidência de dano foi avaliada através de um painel de especialistas que julgaram os atributos relacionados ao método de rastreamento. Tal processo definiu a exclusão de quatro critérios de rastreamanto ("poliestireno de sódio", "C. difficile positivo", "nível de lidocaína > 5ng/ml” e "nível de teofilina $>20 \mu \mathrm{g} / \mathrm{ml}$ "). O poliestireno de sódio, medicamento utilizado para tratamento de hipercalemia, não se encontra registrado para comercialização no Brasil ${ }^{17}$. O uso do critério "Clostridium difficile positivo" para rastreamento de evento adverso a medicamentos se justifica por sua relação com o aparecimento de colite associada ao uso de antibióticos. No entanto, o exame para a detecção das toxinas do C. difficile através da cultura de células não é realizado no hospital estudado. Da mesma forma, a instituição não realiza a mensuração do nível sérico de lidocaína e teofilina.

O critério "sulfato de protamina" foi fundamental para detectar eventos de hemorragia relacionados à administração de heparina. Os rastreadores de "glicose $<50 \mathrm{mg} / \mathrm{dl}$ " e "glicose hipertônica $25 \%$ ou $50 \%$ " mostraram uma boa performance na detecção de hipoglicemia, observada a partir da comparação entre o número de eventos adversos a medicamentos identificados por rastreador específico. No entanto, todos os eventos identificados pelo critério "glicose hipertônica $25 \%$ ou 50\%" também foram rastreados pelo critério "glicose <
$50 \mathrm{mg} / \mathrm{dl}$ ", indicando uma sobreposição dos rastreadores. Como o segundo rastreador identificou mais eventos de hipoglicemia, recomenda-se a utilização desse critério.

O processo de julgamento definiu a modificação de oito critérios ("difenidramina" "droperidol", "glicose sérica < $50 \mathrm{mg} / \mathrm{dl}$ ", "aumento sérico de creatinina, "nível de digoxina > 2ng/ml", "nível de gentamicina ou tobramicina $>10 \mu \mathrm{g} / \mathrm{ml}$ a $>2 \mu \mathrm{g} / \mathrm{ml}$ ", "nível de amicacina $>30 \mu \mathrm{g} / \mathrm{ml}$ a $>10 \mu \mathrm{g} /$ ml" e "nível de vancomicina $>26 \mu \mathrm{g} / \mathrm{ml}$ "). Os rastreadores relacionados aos níveis séricos de aminoglicosídeos e vancomicina apresentaram baixa viabilidade segundo avaliação dos painelistas. No entanto, os possíveis eventos adversos relacionados ao uso desses antibióticos devem ser identificados a partir de sinais e sintomas de intoxicação. Zumbido alto, cefaléia de intensidade moderada, náuseas, vômitos, dificuldade de equilíbrio e aumento de creatinina são os principais sinais e sintomas de intoxicação por aminoglicosídeos ${ }^{18}$. O uso de vancomicina pode ocasionar reações eritematosas ou urticariformes, rubor, taquicardia e hipotensão ${ }^{19}$.

O critério "antieméticos" foi identificado 33 vezes nos prontuários revisados $(\mathrm{n}=112)$, mas não permitiu a identificação de possíveis eventos adversos, fato incomum nesse tipo de investigação. No estudo de Rozenfeld et al..$^{20}$ o critério "antieméticos" foi um dos principais rastreadores identificados, sendo capaz detectar eventos adversos de náuseas e vômitos. A detecção de eventos adversos a medicamentos requer, além da análise prescrição médica, o reconhecimento de possíveis efeitos de outros medicamentos prescritos, assim como a descrição do evento na evolução do paciente no prontuário. Duas situações podem estar presentes: a ausência de registro em prontuário quando o evento é identificado, ou a dificuldade de estabelecimento da relação de causalidade, nos casos em que os sinais e sintomas do quadro clínico do paciente interferem na avaliação.

Dos critérios avaliados foram mantidos onze do IHI ("vitamina K", "flumazenil”, "naloxona", "antidiarréicos", "PTT > 100 segun- 
dos", "INR > 6", "neutrófilos < $3000 \times 10^{6} / \mu \mathrm{l}$ ", "sedação, letargia e queda", "rash", "retirada abrupta do medicamento" e "transferência para Unidade de Terapia Intensiva ou SemiIntensiva"). Entre os prontuários analisados não foram identificados dois dos critérios propostos: "naloxona" e "flumazenil". Sugere-se que o perfil nosológico da unidade estudada, referência na investigação diagnóstica e no tratamento das doenças cardiovasculares, justifica tal fato. Durante a revisão dos prontuários, o critério "antidiarréicos" apareceu apenas uma vez e não esteve associado à identificação de eventos adversos. Acredita-se que a utilização desse critério seja reavaliada em estudos futuros, em função da dificuldade de estabelecimento da relação de causalidade.

A administração de vitamina K permitiu identificar eventos relacionados ao uso excessivo de anticoagulantes orais, como a varfarina. A dose de varfarina é prescrita de acordo com a medida da INR do paciente; quando maior que 6 há aumento do risco para episódios graves de sangramento. Nestes casos, o medicamento é interrompido temporariamente, se necessário, e é administrada vitamina $\mathrm{K}^{15}$.

Os critérios de rastreamento "retirada abrupta do medicamento" e "transferência para a Unidade de Terapia Intensiva ou Semi-Intensiva" representam rastreadores muito sensíveis na detecção de eventos ${ }^{11}$, porém devem ser analisados cuidadosamente, tendo em vista o estabelecimento do nexo causal. Um medicamento pode ser retirado da prescrição de forma abrupta e o paciente encaminhado para uma unidade de maior complexidade, sem que tais fatos possuam qualquer relação com a ocorrência de um evento adverso. No entanto, quando ocorre um evento que ocasionou um dano ao paciente, há uma alta probabilidade de o medicamento ser suspenso. Neste estudo, o critério "retirada abrupta do medicamento" foi o que melhor identificou eventos adversos, assim como foi demonstrado nos achados do $\mathrm{IHI}^{11}$.

O prontuário do paciente é um importante instrumento na atenção à saúde, pois proporciona um registro documentado da assistência prestada pela equipe transdisciplinar. No entanto, o registro nem sempre retrata com clareza o processo assistencial. Assim, a qualidade do registro pode representar uma dificuldade adicional a ser enfrentada no processo de identificação de possíveis eventos adversos. Os critérios de rastreamento devem ser avaliados em diferentes partes do prontuário, dentre elas a folha de internação, as prescrições médicas, as evoluções e os exames laboratoriais, o que torna essa avaliação mais extensa e complexa.

Os registros possuem limitações e falhas que podem comprometer a detecção dos rastreadores identificados a partir das informações descritas nas evoluções, prescrições e exames laboratoriais. Determinados eventos são privilegiados e mais valorizados em relação a outros no momento do registro. Em geral, os eventos que comprometem a vida do paciente são registrados com maior atenção e detalhamento. Todavia, eventos pouco conhecidos e que não comprometem a vida muitas vezes não são registrados. Podemos observar esse fato em relação aos casos de intoxicação por digitálicos. Tal intoxicação é um tipo de evento adverso amplamente conhecido pelos profissionais de saúde, sendo os sinais e sintomas reconhecidos e relatados nos prontuários. No entanto, outros eventos, como, por exemplo, a intoxicação por aminoglicosídeos, podem ocasionar sinais que passem desapercebidos, não sendo portanto registrados.

Neste estudo, a incidência de eventos adversos a medicamentos foi de $14,3 \%$. Em 25\% dos casos houve a necessidade de intervenção para o suporte de vida. Entre os medicamentos envolvidos estão anticoagulantes injetáveis e orais, antiagregantes plaquetários, hipoglicemiantes orais, insulina, digitálicos, diuréticos e contraste radiológico.

A frequência de eventos adversos a medicamentos tem mostrado uma grande variação na literatura em relação à natureza e complexidade de técnicas utilizadas na identificação e na variabilidade das estima- 
tivas de eventos ${ }^{21-29}$. A incidência de eventos adversos mostrou grandes diferenças, variando de $1,3 \%$ a $15,2 \%$.

Um aspecto a ser considerado entre os diferentes estudos é o perfil de gravidade dos casos analisados, ponto crítico a ser observado na comparação entre hospitais, já que, no conjunto, os pacientes podem compor grupos extremamente heterogêneos em relação ao consumo de recursos e aos riscos clínicos. A gravidade dos casos exerce forte influência sobre o resultado do cuidado prestado e tem sido destacada como um importante fator de confundimento.

No estudo realizado pelo $\mathrm{IHI}^{11}$ a taxa de eventos adversos foi medida a partir do número de doses aplicadas do medicamento, variando entre 2,47 a 4,81 por 1.000 doses $^{11}$. No entanto, tais resultados não podem ser comparados com os obtidos nessa pesquisa, pois a taxa de eventos adversos foi estabelecida a partir do número de casos avaliados.

No Rio de Janeiro, dois importantes estudos identificaram a ocorrência de eventos adversos a medicamentos, com frequências entre $5,5 \%^{29-31}$ e $15,6 \%{ }^{20}$. O estudo de Rozenfeld et al. ${ }^{20}$ utilizou-se de critérios rastreadores muito próximos aos utilizados nesse estudo e os eventos adversos estiveram relacionados com a administração de analgésicos, hipoglicemiantes, antiinflamatórios e anticoagulantes. O evento adverso a medicamento deve ser entendido como um evento sentinela, sendo fundamental a compreensão de como e do porquê de sua ocorrência. A utilização dos critérios de ras- treamento para evento adverso possibilita a identificação dos tipos mais incidentes, bem como permite a implantação de estratégias para evitá-los e a elaboração de um plano de metas da instituição de saúde para sua redução.

\section{Conclusão}

O sistema de vigilância de eventos adversos a medicamento necessita de bons rastreadores para identificar possíveis eventos. A detecção destes possibilita intervenções rápidas, bem como a prevenção de futuros eventos e garantia da segurança do paciente.

Os critérios de rastreamento de eventos adversos a medicamento possibilitam um monitoramento dos eventos ao longo do tempo. Desta forma permitem avaliar se as estratégias implementadas no sistema de medicação resultaram em melhoria na qualidade da assistência, traduzida em uma maior segurança para os pacientes e na redução de eventos adversos a medicamento. Estes critérios poderão ser indicados para o desenvolvimento de outros estudos relacionados com essa temática, a fim de se conhecer a incidência de eventos adversos nos hospitais brasileiros.

A implantação de rotinas de seguimento dos rastreadores utilizados neste estudo e a revisão do sistema de medicação da instituição estudada poderão favorecer o monitoramento e a implementação de mecanismos de defesa, barreira e proteção voltadas para a melhoria da segurança do paciente.

\section{Referências}

1. Thomas EJ, Petersen LA. Measuring errors and adverse events in health care. J Gen Med 2003; 18: 61-7.

2. Leape LL, Kabcenell AI, Gandhi TK, Carver P, Nolan TW, Berwick DM. Reducing adverse drug events: lessons from a breakthroughs series collaborative. Jt Comm J Qual Improv 2000; 26(6): 321-31.

3. Coimbra JAH, Cassiani SHB. Nursing responsibility in the administration of medication: some reflections for a safe practice with quality. Rev Latino-am Enfermagem 2001; 9(2): 56-60.
4. World Health Organization. WHO Department of Essential Drugs and Medicines. The Uppsala Monitoring Centre. The importance of pharmacovigilance: safety monitoring of medicinal/products. Genebra; 2002.

5. Mills DH. Medical insurance feasibility study: a technical summary. West J Med 1978; 128: 360-5.

6. Leape LL, Brennan TA, Laird N, Lawthers AG, Localio $\mathrm{AR}$, Barnes BA et al. The nature of adverse events in hospitalized patients. Results of the Harvard Medical Practice Study II. N Engl J Med 1991; 324(6): 377-84. 
7. Gawande AA, Thomas E, Zinner MJ, Brennan TA. The incidence and nature of surgical adverse events in Colorado and Utah in 1992. Surgery 1999; 126(1): 66-75.

8. Wilson RM, Runciman WB, Gibbert RW, Harrison BT, Newby L, Hamilton JD. The quality in australian health care study. Med J Aust 1995; 163: 458-71.

9. Davis P, Lay-Yeer R, Scoot A, Briant R, Schug S. Acknowledgement of a "no fault" medical injury review of patients hospital records in New Zealand. Br Med J 2003; 326: 79-80.

10. Vicent C, Neale G, Woloshynowych M. Adverse events in british hospitals: preliminary retrospective record review. Br Med J 2001; 322: 517-9.

11. Rozich JD, Haraden CR, Resar RK. Adverse drug event trigger tool: a pratical methodology for measuring medication related harm. Qual Saf Health Care Med [serial on the Internet]. 2003. Disponível em http:// www.ihi.org/ihi/uploads/AdverseDrugEventTriggerTool. pdf. [Acessado em 15 mai. 2007]

12. National Coordinating Council for Medication Error Reporting and Prevention [homepage na Internet]. Rockville: Taxonomy of medication errors. Disponível em http://www.nccmerp. org/aboutMedErrors.html. [Acessado em 10 abr. 2007]

13. Brasil. Ministério da Saúde. Agência Nacional de Vigilância Epidemiológica [homepage na Internet]. Brasília: Hospital Sentinela 2003. Disponível em http:// www.anvisa.gov.br/ servicosaude/hsentinela/index.ht. [Acessado em 12 set. 2008]

14. Wright JTC, Giovinazzo, RA. Delphi: uma ferramenta de apoio no planejamento prospectivo. Cad Pesq Adm 2000; 1(12): 54-65.

15. Majerus PW, Tollefsen DM. Coagulação sanguínea e anticoagulants, trombolíticos e fármacos antiplaquetários. In: Bruton LL, Lazo JS, Parker KL. Goodman \& Gilman: as bases farmacológicas da terapêutica. Rio de Janeiro: McGraw-Hill; 2006. p. 132139.

16. Davis SN. Insulina, agentes hipoglicemiantes orais e farmacologia do pâncreas endócrino. In: Bruton LL, Lazo JS, Parker KL. Goodman \& Gilman: as bases farmacológicas da terapêutica. Rio de Janeiro: McGraw-Hill; 2006. p. 1459-88.

17. Brasil. Ministério da Saúde. Relação Nacional de Medicamentos Essenciais - Rename. Brasília: Ministério da Saúde; 2008.

18. Chambers HF. Aminoglicosídios. In: Bruton LL, Lazo JS, Parker KL. Goodman \& Gilman: as bases farmacológicas da terapêutica. Rio de Janeiro: McGraw-Hill; 2006. p. 1039-54.

19. Henry FC. Inibidores da síntese de proteínas e agentes antibacterianos diversos. In: Bruton LL, Lazo JS, Parker KL. Goodman \& Gilman: as bases farmacológicas da terapêutica. Rio de Janeiro: McGraw-Hill; 2006. p. 105581.
20. Rozenfeld S, Chaves SMC, Reis LGC, Martins M, Travassos C, Mendes W et al. Efeitos adversos a medicamentos em um hospital público: estudo piloto. Rev Saúde Pública 2008; 43(5): 887-90.

21. Suh DC, Woodall BS, Shin SK, Hermes-De Santis ER. Clinical and economic impact of adverse drug reactions in hospitalized patients. Ann Pharmacother 2000; 34: 1373-9.

22. Senst BL, Achusim LE, Genest RP, Cosentino LA, Ford CC, Little JA, et al. Practical approach to determining costs and frequency of adverse drug events in a health care network. Am J Health Syst Pharm 2001; 58: 1126-32.

23. Corral-Baena S, Guerrero-Aznar MD, Beltrán-García M, Salas-Turrens J. Utilización del CMBD como herramienta para la detección de acontecimientos adversos a medicamentos. Farm Hosp 2004; 28: 258-65.

24. Cohen MM, Kimmel NL, Benage MK, Cox MJ, Sanders $\mathrm{N}$, Spence D, et al. Medication safety program reduces adverse drug events in a community hospital. Qual Saf Health Care 2005; 14: 169-74.

25. Mycyk MB, McDaniel MR, Fotis MA, Regalado J. Hospitalwide adverse drug events before and after limiting weekly work hours of medical residents to 80 . Am J Health Syst Pharm 2005; 62: 1592-5.

26. Kilbridge PM, Campbell UC, Cozart HB, Mojarrad MG. Automated surveillance for adverse drug events at a community hospital and an academic medical center. $J$ Am Med Inform Assoc 2006; 13: 372-7.

27. Bond CA, Raehl CL. Clinical pharmacy services, pharmacy staffing, and adverse drug reactions in United States hospitals. Pharmacotherapy 2006; 26: 735-47.

28. Hougland P, Xu W, Pickard S, Masheter C, Williams SD. Performance of International Classification of Diseases, $9^{\text {th }}$ revision, clinical modification codes as an adverse drug event surveillance system. Med Care 2006; 44: 62936.

29. Lugardon S, Desboeuf K, Fernet P, Montastruc JL, Lapeyre-Mestre M. Using a capture-recapture method to assess the frequency of adverse drug reactions in a French university hospital. Br J Clin Pharmacol 2006; 62: 225-31.

30. Mendes W, Travassos C, Martins M, Noronha JC de. Revisão dos estudos de avaliação da ocorrência de eventos adversos em hospitais. Rev Bras Epidemiol 2005; 8(4): 393-406.

31. Mendes W, Travassos C, Martins M, Marques PM. Adaptação dos instrumentos de avaliação de eventos adversos para uso em hospitais brasileiros. Rev Bras Epidemiol 2008; 11(1): 55-66.

Recebido em: 12/09/09 Versão final reapresentada em: 18/07/10 Aprovado em: 09/08/10 